\title{
Extra-terrestrial Geophysics to Understand Climate Change and Open Access Journal: Journal of Geophysics and Remote Sensing
}

\section{Saumitra Mukherjee*}

School of Environmental Sciences Jawaharlal Nehru University, New Delhi, India

Climate change has been addressed by scientific studies based on the influence of human activities like production of industrial effluents, land use changes and other activities due to development of the society. It has been suggested that suggests that the carbon in atmosphere was reduced in geological past 470 million years ago by the advent of plant species on the earth. All these variables might have influenced the environment of the earth leading to the climate change but the process is slow in comparison to the extra terrestrial influence. An attempt can be made to understand the influence of extraterrestrial geophysical activities as one of the important factors of climate change. The influence of Sun and distant stars on the environment can be studied during the cyclic changes in the Sun as well as episodic changes in the environment due to the effect of other celestial objects in between SunEarth environment. It is evident that if a short term changes in the sunearth weather due to eclipse can influence the environment of the earth temporarily it can be a possible key of climate change due to abnormal solar behaviour. Recent researches has projected the change in earths ecosystem due to climate change which needs to be reconsidered based on the parameters of changing extra terrestrial activities of Sun-Earth system. The effects of eclipse were observed in a part of China on $23^{\text {rd }}$ July 2009 which shows that the cosmic ray intensity decreases with the decrease in Electron flux recorded by the Sun Observatory Heliospheric Observatory (SOHO) satellite. The attempt was based on the changes in the concentration of atmospheric gases like Sulpher Dioxide, Nitrogen Dioxide, Aerosol and Cloud cover. Research shows direct correlation of cosmic ray intensity, heliopysical and atmospheric variation during the solar eclipse. It will be useful educational information to understand the atmosphere in variable condition.

It is not possible to study the atmospheric changes in isolation. It has been inferred that there are indirect links exists in between the changes within the Sun with the thermosphere, ionosphere, atmosphere and Geosphere. The geophysical variables in between Sun and Earth as well as within earth's different layers can be inferred by using remote sensing and geophysical devices. Earth's geophysical parameters like resistivity, gravity, magnetic and seismic variations can be correlated effectively with the changes in the earth's magnetic field, electron flux, proton flux and incoming cosmic ray count rate changes. After more than a century of controversy, the debate as to whether solar variability has any significant effect on the climate of the Earth remains to be settled. Present work attempts to establish a new hypothesis on Star-Sun-Earth atmospheric interactions and opens a new horizon of near accurate climate change prediction research.
*Corresponding author: Saumitra Mukherjee, Professor (Geology \& Remote sensing), School of Environmental Sciences Jawaharlal Nehru University, New Delhi, India, Tel: 91-11-26704312; Fax: 91-11-26704312; E-mail: saumitra@mail.jnu.ac.in

Received February 10, 2014; Accepted February 11, 2014; Published February 15,2014

Citation: Mukherjee S (2014) Extra-terrestrial Geophysics to Understand Climate Change and Open Access Journal: Journal of Geophysics and Remote Sensing. J Geophys Remote Sensing 3: e109. doi:10.4172/2169-0049.1000e109

Copyright: (๑) 2014 Mukherjee S. This is an open-access article distributed under the terms of the Creative Commons Attribution License, which permits unrestricted use, distribution, and reproduction in any medium, provided the original author and source are credited. 